\section{Harnsäuresenkung schützt Herz und Gefäße}

_ Urikostatika fallen bei der Kontrolle der Medikamentenlisten älterer Patienten oft als erste dem "Rotstift" zum Opfer. Dies kann schwerwiegende Konsequenzen haben, denn Harnsäure ist ein unabhängiger kardiovaskulärer Risikofaktor, der eine treibende Kraft für Hypertonie und Nephropathie ist.

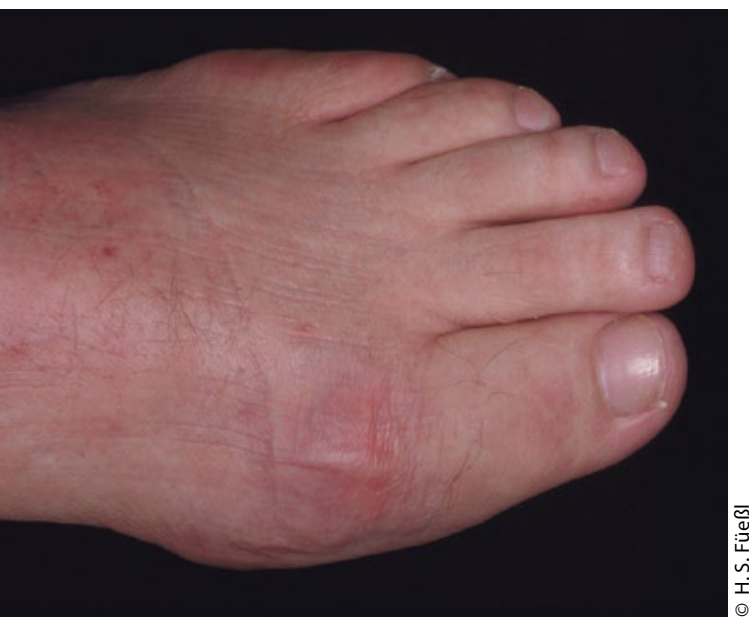

Zu viel Harnsäure schadet nicht nur den Gelenken, sondern auch dem Herzen.
Harnsäure ist beim Menschen das Endprodukt des Purinstoffwechsels. Mit steigenden Konzentrationen kommt es zur Ausfällung von Uratkristallen im Gewebe. Die Folge: Schmerzhafte Gichtanfälle. „Die typischen Ablagerungen betreffen aber nicht nur die Gelenke, sondern auch die Gefäße", erklärte Prof. Claudio Borghi, Chefarzt der Klinik für Innere Medizin der Universität Bologna und Leiter der Abteilung für Hypertonie-Forschung und der HerzinsuffizienzAmbulanz, Bologna (Italien). Es kommt zur endothelialen Dysfunktion mit Abnahme der vasodilatativen Kapazität und Zunahme der Gefäßsteifigkeit. Dies erklärt die enge Assoziation zwischen Hypertonie und $\mathrm{Hy}$ perurikämie.

Klinische und experimentelle Daten sprechen dafür, dass die Hyperurikämie eine kausale Rolle bei der Entwicklung von $\mathrm{Hy}$ pertonie und/oder Nierenfunktionsstörungen spielt. Dabei steigt das kardiovaskuläre Risiko mit zunehmenden Harnsäurespiegeln. Die Schwelle für ein erhöhtes kardiovaskuläres Risiko scheint deutlich niedriger zu liegen als die Schwelle für das Auftreten von Gicht. „Die pharmakologische Sen- kung des Harnsäurespiegels sollte frühzeitig in Erwägung gezogen werden", betonte Borghi.

First-line-Option bei der Harnsäuresenkung sind die Xanthinoxidasehemmer Allopurinol und Febuxostat (Adenuric ${ }^{\circledR}$ ). Prof. Thomas Bardin, Leiter der Abteilung für Rheumatologie am Hôpital Lariboisière, Paris (Frankreich), verwies auf die CONFIRMSStudie, in der die Wirksamkeit beider Urikostatika verglichen wurde. Teilnehmer waren 2269 Gichtpatienten, von denen $65 \%$ eine eingeschränkte Nierenfunktion und damit ein hohes kardiovaskuläres Risiko aufwiesen. $71,6 \%$ der mit Febuxostat, aber nur 42,3\% der mit Allopurinol behandelten $\mathrm{Pa}$ tienten erreichten den Harnsäurezielwert $<6 \mathrm{mg} / \mathrm{dl}$.

Im Gegensatz zu Allopurinol muss für Febuxostat keine Dosisanpassung bei leichter Niereninsuffizienz erfolgen, und das Interaktionspotenzial ist geringer. Auch wurden bisher unter Febuxostat keine der unter Allopurinol gefürchteten lebensbedrohlichen Hautreaktionen beobachtet.

Dr. Kirsten Westphal

Minisymposium beim 23. European Meeting on Hypertension \& Cardiovascular Protection, Mailand, 14.-17. Juni 2013 (Veranstalter: Menarini)

\section{Schlaganfallprophylaxe mit Stöpsel im Herzohr}

_ Der Ansatz ist plausibel: Da die meisten Thromben bei Vorhofflimmern im linken Herzohr entstehen, müsste ein Verschluss dieses Herzanhängsels die Schlaganfallrate senken. Erste Studien mit dem WatchmanDevice bestätigen dieses Konzept.

Das Watchman-System besteht aus einem Schirmchen, aufgespannt in einem selbstexpandierenden Nitinol-Rahmen, der mit Häkchen versehen ist, um das Implantat zu fixieren. Der Applikationskatheter wird transseptal in das linke Vorhofohr geführt. In der PROTECT-AF-Studie (Vergleich Device versus Warfarin) zeigte sich, wie wichtig die Erfahrung des Untersuchers für das Ergebnis ist. Wegen einer relativ hohen Rate von Perikardergüssen und prozedurassoziierten Schlaganfällen verweigerte die FDA zunächst die Zulassung, während in Europa das Konzept offenbar überzeugte.

Die anschließende PREVAIL-Studie sollte mit einem verbesserten Trainingsprogramm Klarheit bringen. Sie umfasste 407 Patien- ten mit Vorhofflimmern (CHADS-Score im Schnitt 2,6) aus 41 US-Zentren. Patienten mit "Stöpsel" erhielten nach der Implantation 45 Tage lang ein Antikoagulans (Warfarin). Einige wichtige Ergebnisse:

_ Die prozedurale Erfolgsrate konnte von 90,9\% (PROTECT-AF) auf 95,1\% in PREVAIL gesteigert werden.

- Den ersten primären Sicherheitsendpunkt erreichten sechs von 269 Patienten (2,2\%). Dieser Endpunkt umfasste Tod, ischämische Embolie und prozedur- oder devicebezogene Komplikationen mit kardiovaskulärer oder endovaskulärer Intervention innerhalb von sieben Tagen nach der Intervention.

_ Vaskuläre Komplikationen nahmen signifikant ab: von $8,7 \%$ in PROTECT-AF auf $4,4 \%$ in PREVAIL.

— Der zweite primäre Endpunkt betraf die 18-Monats-Effektivität und gab Anlass zu Diskussionen. Die Ereignisraten in beiden Gruppen waren ähnlich, eine definitive
Analyse fehlt noch, weil zu wenig Patienten die 18-Monats-Grenze erreicht hatten. Vor allem aber fiel die extrem niedrige Ereignisrate in der Warfaringruppe $(0,7$ pro 100 Patientenjahre) auf. In der ROCKET-AF-Studie hatte die Kontrollgruppe eine Ereignisrate von 2,2 pro 100 Patientenjahre.

Die bisherige Datenlage fasste Priv.-Doz. Dr. med. Peter Sick, Klinik für Kardiologie in Regensburg, so zusammen: Trotz Implantation des Watchman-Devices bei Patienten mit höherem Risiko war die Implantation auch bei neuen Untersuchern sicher. Das Trainingsprogramm war also erfolgreich. Und die Daten der PREVAIL-Studie bestätigen den früher publizierten Nutzen dieses Konzepts, die Schlaganfallrate bei Hochrisikopatienten zu senken.

Dr. Jochen Aumiller

Meet the Expert, Jahrestagung der Deutschen Gesellschaft für Kardiologie, Mannheim, 4.4.2013 (Veranstalter: Boston Scientific) 